\title{
Prone positioning for non-intubated hypoxaemic patients with COVID-19: cheap, easy and makes sense, but does it work?
}

\author{
Eric D. Morrell $\mathbb{1}^{1,2,3}$ and Mark M. Wurfel ${ }^{2}$
}

${ }^{1}$ VA Puget Sound Heath Care System, Seattle, WA, USA. ${ }^{2}$ Harborview Medical Center, Seattle, WA, USA. ${ }^{3}$ Division of Pulmonary, Critical Care, and Sleep Medicine, University of Washington Medical Center, University of Washington, Seattle, WA, USA.

Corresponding author: Eric D. Morrell (edmorrel@uw.edu)

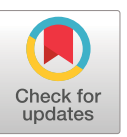

Copyright @The authors 2022

This version is distributed under the terms of the Creative Commons Attribution Non-Commercial Licence 4.0. For commercial reproduction rights and permissions contact permissions@ersnet.org

Received: 5 Sept 2021 Accepted: 20 Sept 2021

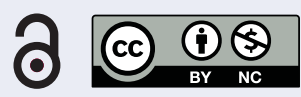

Shareable abstract (@ERSpublications)

This editorial evaluates a large observational study that identified strong associations between awake prone positioning and decreased mortality in hospitalised patients with COVID-19 https://bit. ly $/ 3 \mathrm{~m} 3 \mathrm{NeAx}$

Cite this article as: Morrell ED, Wurfel MM. Prone positioning for non-intubated hypoxaemic patients with COVID-19: cheap, easy and makes sense, but does it work? Eur Respir J 2022; 59: 2102416 [DOI: 10.1183/13993003.02416-2021].
Since the 1970s, prone positioning has been proposed as an inexpensive and physiologically justified management strategy for patients with acute respiratory distress syndrome (ARDS). The physiological rationale for prone positioning in ARDS has been well described [1, 2]. Placing a person in the prone position reduces lung compression, which facilitates better ventilation/perfusion matching and leads to improved oxygenation. However, determining whether patients receive clinically meaningful benefit from prone positioning in the form of reduced mortality or time supported on mechanical ventilation has been challenging. Early trials that used prone positioning as a "rescue" therapy or applied relatively small "doses" of prone positioning (i.e. limited duration of time in prone position) did not demonstrate improvements in mortality or ventilator-free days (VFDs) [3-5]. The landmark PROSEVA trial published in 2013 enrolled patients with at least moderate ARDS (arterial oxygen tension to fraction of inspired oxygen ratio <150) who were early in their hospital course ( $<36 \mathrm{~h}$ after intubation) and then randomised them to standard care alone (including low tidal volume ventilation) or at least $16 \mathrm{~h}$ per day of prone positioning [6]. In contrast to prior trials, PROSEVA identified a marked reduction in mortality and VFDs in ARDS patients treated with prone positioning. There have been many proposed explanations for PROSEVA's success after repeated negative results in earlier trials of proning. However, selection of a more severe patient population early in their disease clearly differentiated PROSEVA from prior trials. A major question that remained after PROSEVA was whether there might be a broader set of patients with hypoxaemic respiratory failure that might benefit from prone positioning. The onset of the coronavirus disease 2019 (COVID-19) pandemic has forced us to ask whether non-intubated hypoxaemic patients infected with SARS-CoV-2 might represent an additional group of patients that might benefit from prone positioning.

The COVID-19 pandemic has dramatically increased the number of patients with moderate-to-severe ARDS who are treated outside of intensive care units [7]. There is an urgent need to mitigate escalation in respiratory support and decrease rates of intubation, particularly in healthcare systems where the volume of COVID-19 patients exceeds hospital capacity. Awake prone positioning for non-intubated patients with hypoxaemia and COVID-19 has been associated with improved oxygenation and decreased rates of intubation in some [8-15], but not all, reports [16-20]. None of these previously published observational studies included more than $\mathrm{n}=200$ subjects nor accounted for factors that could contribute to selection bias, such as age or oxygen delivery device. A recent randomised meta-trial of COVID-19 patients supported on high-flow nasal cannula (HFNC) demonstrated decreased rates of treatment failure and subsequent intubation in patients treated with awake prone positioning versus standard care; however, there was no difference in 28-day mortality [21]. The impact of awake prone positioning on non-intubated COVID-19 patients supported on low-flow and other non-HFNC oxygen delivery devices has not been tested in an interventional trial. 
In this issue of the European Respiratory Journal, Perez-Nieto et al. [22] present findings from the APPONOX study that suggest prone positioning of awake, non-intubated, patients with hypoxaemia due to COVID-19 is associated with decreased rates of subsequent intubation and mortality. This large retrospective cohort study included patients $(n=827)$ from 27 hospitals in Mexico and Ecuador who were infected with SARS-CoV-2 and were hypoxaemic (peripheral capillary oxygen saturation <94\%). Only $10 \%$ of patients were treated with HFNC at time of enrolment, with the remainder supported on low-flow nasal cannula (49\%) or non-rebreather mask (41\%). The authors compared patients who were treated for greater than $2 \mathrm{~h}$ of prone positioning (AP group; $\mathrm{n}=505$ ) versus subjects in whom prone positioning was not attempted or were treated for less than $2 \mathrm{~h}$ (AS group; awake supine; $n=322$ ). In the AP group, the median time to initiation of proning after hospital arrival was $15 \mathrm{~h}$ and patients were proned for a median time of $12 \mathrm{~h}$ during their hospital course. The primary outcome for the study was rates of intubation. In order to mitigate confounding by indication, the authors performed a propensity score analysis including variables such as age, degree of hypoxaemia, supplemental oxygen delivery device, comorbidities, treatment with steroids, and acuity of care. The authors found that $23.6 \%$ of AP patients were subsequently intubated versus $40.4 \%$ of AS patients (adjusted OR 0.50, 95\% CI $0.36-0.71 ; \mathrm{p}<0.0001$ after propensity analysis). Mortality was also significantly lower in the AP versus AS group (19.8\% versus 37.3\%).

The major strengths of this study include the relatively large size of the cohort, the rigor of the analyses, which included propensity score matching, and the diversity of the hospitals and patients included in the study. $90 \%$ of patients in this study were not supported on HFNC, suggesting that awake prone positioning of non-intubated hypoxaemic patients with COVID-19 might be beneficial to a broader population beyond those supported by this scarce respiratory support device [21]. Indeed, as the authors note, this study was conducted at some sites that dealt with hospital strain and limited resources [23]. The findings from this study may be generalisable to institutions where scarcity of advanced respiratory care resources has a major influence on patient outcomes [24].

Though encouraging, the results of this study should be interpreted with caution. The authors included multiple analyses to address indication and treatment biases; however, given the highly complex and rapidly evolving nature of care for hypoxaemic COVID-19 patients, it is not unlikely that some degree of residual confounding exists. For example, it is possible patients who did not receive awake prone positioning were too frail or had some other factors limiting appropriateness or feasibility of prone positioning, and these factors could also have increased risk for intubation. The disparity in the proportion of patients receiving HFNC and non-rebreather masks between the AP versus AS groups could have confounded the results given the potential beneficial aspects of HFNC treatment in COVID-19 [25, 26]. The authors importantly adjusted for this in their analyses, and the proportion of patients receiving HFNC was only $10 \%$ in the entire cohort. Finally, although the authors found an association between awake prone positioning and decreased mortality, the safety of proning was not assessed. A recent trial of prone positioning in COVID-19 patients supported on HFNC did not demonstrate a concerning signal for harm [21]. Additional studies testing whether proning non-intubated patients might inappropriately delay initiation of invasive mechanical ventilation and lead to worse overall outcomes are currently underway (ClinicalTrials.gov NCT04347941, NCT04344587, NCT04325906, NCT04358939 and NCT04395144) [2].

In conclusion, the APPONOX study confirms that awake proning of non-intubated hypoxaemic patients with COVID-19 holds promise as a cheap, easy, and effective therapy for reducing rates of intubation and mortality in hypoxaemic patients with COVID-19. Reducing rates of intubation may be particularly important in healthcare systems where access to advanced respiratory care resources is limited. There are multiple ongoing randomised controlled trials underway to confirm the findings from this study. Until the results from these studies are published, it seems reasonable to encourage non-intubated hypoxaemic patients with COVID-19 to assume a prone position.

Conflict of interest: None declared.

Support statement: This work was supported by the National Heart, Lung, and Blood Institute (grant K23 HL144916). Funding information for this article has been deposited with the Crossref Funder Registry.

\section{References}

1 Scholten EL, Beitler JR, Prisk GK, et al. Treatment of ARDS with prone positioning. Chest 2017; 151: 215-224.

2 Touchon F, Trigui Y, Prud'homme E, et al. Awake prone positioning for hypoxaemic respiratory failure: past, COVID-19 and perspectives. Eur Respir Rev 2021; 30: 210022.

3 Guerin C, Gaillard S, Lemasson S, et al. Effects of systematic prone positioning in hypoxemic acute respiratory failure: a randomized controlled trial. JAMA 2004; 292: 2379-2387. 
4 Gattinoni L, Tognoni G, Pesenti A, et al. Effect of prone positioning on the survival of patients with acute respiratory failure. N Engl J Med 2001; 345: 568-573.

5 Taccone P, Pesenti A, Latini R, et al. Prone positioning in patients with moderate and severe acute respiratory distress syndrome: a randomized controlled trial. JAMA 2009; 302: 1977-1984.

6 Guérin C, Reignier J, Richard J-C, et al. Prone positioning in severe acute respiratory distress syndrome. N Engl J Med 2013; 368: 2159-2168.

7 Coopersmith CM, Antonelli M, Bauer SR, et al. The surviving sepsis campaign: research priorities for coronavirus disease 2019 in critical illness. Crit Care Med 2021; 49: 598-622.

8 Thompson AE, Ranard BL, Wei Y, et al. Prone positioning in awake, nonintubated patients with COVID-19 hypoxemic respiratory failure. JAMA Intern Med 2020; 180: 1537-1539.

9 Damarla M, Zaeh S, Niedermeyer S, et al. Prone positioning of nonintubated patients with COVID-19. Am J Respir Crit Care Med 2020; 202: 604-606.

10 Caputo ND, Strayer RJ, Levitan R. Early self-proning in awake, non-intubated patients in the emergency department: a single ED's experience during the COVID-19 pandemic. Acad Emerg Med 2020; 27: 375-378.

11 Sartini C, Tresoldi M, Scarpellini P, et al. Respiratory parameters in patients with COVID-19 after using noninvasive ventilation in the prone position outside the intensive care unit. JAMA 2020; 323: 2338-2340.

12 Coppo A, Bellani G, Winterton D, et al. Feasibility and physiological effects of prone positioning in non-intubated patients with acute respiratory failure due to COVID-19 (PRON-COVID): a prospective cohort study. Lancet Respir Med 2020; 8: 765-774.

$13 \mathrm{Ng} \mathrm{Z}$, Tay WC, Ho CHB. Awake prone positioning for non-intubated oxygen dependent COVID-19 pneumonia patients. Eur Respir J 2020; 56: 2001198.

14 Prud'homme E, Trigui $\mathrm{Y}$, Elharrar $\mathrm{X}$, et al. Effect of prone positioning on the respiratory support of nonintubated patients with COVID-19 and acute hypoxemic respiratory failure: a retrospective matching cohort study. Chest 2021; 160: 85-88.

15 Ponnapa Reddy M, Subramaniam A, Afroz A, et al. Prone positioning of nonintubated patients with coronavirus disease 2019 - a systematic review and meta-analysis. Crit Care Med 2021; 49: e1001-e1014.

16 Ferrando C, Mellado-Artigas R, Gea A, et al. Awake prone positioning does not reduce the risk of intubation in COVID-19 treated with high-flow nasal oxygen therapy: a multicenter, adjusted cohort study. Crit Care 2020; 24: 597.

17 Padrão EMH, Valente FS, Besen BAMP, et al. Awake prone positioning in COVID-19 hypoxemic respiratory failure: exploratory findings in a single-center retrospective cohort study. Acad Emerg Med 2020; 27: 1249-1259.

18 Elharrar X, Trigui Y, Dols A-M, et al. Use of prone positioning in nonintubated patients with COVID-19 and hypoxemic acute respiratory failure. JAMA 2020; 323: 2336-2338.

19 Johnson SA, Horton DJ, Fuller MJ, et al. Patient-directed prone positioning in awake patients with COVID-19 requiring hospitalization (PAPR). Ann Am Thorac Soc 2021; 18: 1424-1426.

20 Pavlov I, He H, McNicholas B, et al. Awake prone positioning in non-intubated patients with acute hypoxemic respiratory failure due to COVID-19. Respir Care 2022; 67: 102-114

21 Ehrmann S, Li J, Ibarra-Estrada M, et al. Awake prone positioning for COVID-19 acute hypoxaemic respiratory failure: a randomised, controlled, multinational, open-label meta-trial. Lancet Respir Med 2021; 9: P1387P1395.

22 Perez-Nieto OR, Escarraman-Martinez D, Guerrero-Gutierrez MA, et al. Awake prone positioning and oxygen therapy in patients with COVID-19: the APRONOX study. Eur Respir J 2022; 59: 2100265.

23 Fowler Z, Moeller E, Roa L, et al. Projected impact of COVID-19 mitigation strategies on hospital services in the Mexico City Metropolitan Area. PLoS ONE 2020; 15: e0241954.

24 Bravata DM, Perkins AJ, Myers LJ, et al. Association of intensive care unit patient load and demand with mortality rates in US Department of Veterans Affairs Hospitals during the COVID-19 pandemic. JAMA Netw Open 2021; 4: e2034266.

25 Demoule A, Vieillard Baron A, Darmon M, et al. High-flow nasal cannula in critically ill patients with severe COVID-19. Am J Respir Crit Care Med 2020; 202: 1039-1042.

26 Guy T, Créac'hcadec A, Ricordel C, et al. High-flow nasal oxygen: a safe, efficient treatment for COVID-19 patients not in an ICU. Eur Respir J 2020; 56: 2001154. 